Annls Limnol. 16 (1) $1980: 77-89$.

\title{
INCIDENCES DE LA POLLUTION ORGANIQUE ET DE L'EUTROPHISATION SUR LA FAUNE ICHTYOLOGIQUE DE LA SEMOIS
}

\author{
par J. C. PHILIPPART ${ }^{1}$
}

Des inventaires ichtyologiques (pêche à l'électricité) réalisés en 1977 dans la Semois (affluent franco-belge de la Meuse) mettent en évidence les incidences d'une pollution organique chronique (Haute Semois) et d'une eutrophisation grave (Basse-Semois) sur l'ichtyofaune d'un rivière de la Zone à Barbeau :

- élimination ou réduction des populations de $C$. gobio, $S$. trutta, A. bipunctatus, $B$. barbus, $C$. nasus:

- remplacement de $B$. barbus par L. cephalus;

- diminution de la biomasse relative du groupe $B$. barbus $+C$. nasus qui constitue un bon indicateur de l'équilibre structurel de l'ichtyocénose;

- diminution de la biomasse totale de l'ichtyocénose.

Ces résultats font apparaître certains aspects de la polluo-sensibilité (nature écophysioogique) et la résilience (nature démographique) différentielles des espèces de poissons caractéristiques de ce type de rivière.

\section{The effects of organic pollution and eutrophication on the fish fauna of the river Semois.}

Fish surveys by electrofishing in the Semois (a tributary of the river Meuse) in 1977 showed the effects of a marked organic pollution (upper reaches of the Semois) and a serious eutrophication (lower reaches) on the fish fauna in the barbel-zone of the river. There was:

- elimination or reduction of populations of C. gobio, S. trutta, A. bipunctatus, $B$. barbus, C. nasus;

- replacement of B. barbus by L. cephalus;

- reduction of the relative biomass of the group, B. barbus $+C$. nasus, which is a good indicator of the structural stability of the fish-coenosis;

- reduction of the total biomass of fish.

These results illustrate certain aspects of differences in pollution sensitivity (of an ecophysiological nature) and resilience (of a demographic nature) between fish species that are typical of this type of river.

\section{INTRODUCTION}

Les recherches sur la structure et l'écologie des communautés ichtyologiques des rivières de la "Zone à Barbeau " (Huet, 1949) se sont développées en Europe au cours des quinze dernières années.

1. Chercheur qualifié du Fonds National de la Recherche Scientifique (FNRS). institut de Zoologie de l'Université de Liège. Service d'Ethologie et Psychologie animales. Aquarium (Prof. J. C. Ruwet). Unité de Recherches Piscicoles, 22, Quai Van Beneden, B 4020 Liège, Belgique. 
Citons les travaux de Penczak (1972), Penczak et Zalewski (1974), Kolder et coll. (1974) et Penczak et coll. (1976) en Pologne, de Seldlar (1969), Lusk $(1973,1977)$ et Holcik et Bastl (1976) en Tchécoslovaquie, de Huet et Timmermans $(1963,1967)$ et Micha (1971) en Belgique et de Roux (1976) en France. Dans ce même courant s'inscrit notre contribution (Philippart $1977 \mathrm{a}, \mathrm{b}$ ) à l'écologie des populations de poissons dans la Zone à Barbeau de l'Ourthe. En prolongement de cette recherche furent effectués en 1977 des inventaires ichtyologiques dans la Semois, une "rivière à barbeau » franco-belge étudiée antérieurement (1959) dans son cours inférieur par Huet et Timmermans (1963). Le but du présent travail est de décrire la faune ichtyologique de l'ensemble de la Semois en précisant la zonation des poissons et en analysant l'incidence sur l'ichtyofaune des phénomènes de pollution organique et d'eutrophisation qui affectent gravement la rivière.

\section{1. - LA SEMO!S ET LeS StATIONS ETUdiees}

La Semois (fig. 1) prend sa source au sud d'Arlon (alt. : $113 \mathrm{~m}$ ) et se jette dans la Meuse à Monthermé (alt. : $141 \mathrm{~m}$ ) après un parcours de $210 \mathrm{~km}$ (bassin versant $=1358 \mathrm{~km}^{2}$ ) dont $191 \mathrm{~km}$ en Belgique. A la frontière franco-belge, ses caractéristiques hydrologiques pour la décennie 1954-1963 sont (Vereerstraeten 1977) : débit moyen annuel: $29 \mathrm{~m}^{3} / \mathrm{sec}$., début mensuel moyen minimum de juillet: $11 \mathrm{~m}^{3} / \mathrm{sec}$, débit médian : $21 \mathrm{~m}^{3} / \mathrm{sec}$. La Semois traverse deux régions lithologiquement très contrastées: la Lorraine belge (roches alcalines) de la source à Chiny $( \pm 60 \mathrm{~km})$ et l'Ardenne (roches acides) jusqu'au confluent avec la Meuse; cette particularité se répercute à d'autres niveaux : morphologie du cours et surtout pente $(0,73 \%$ en Semois

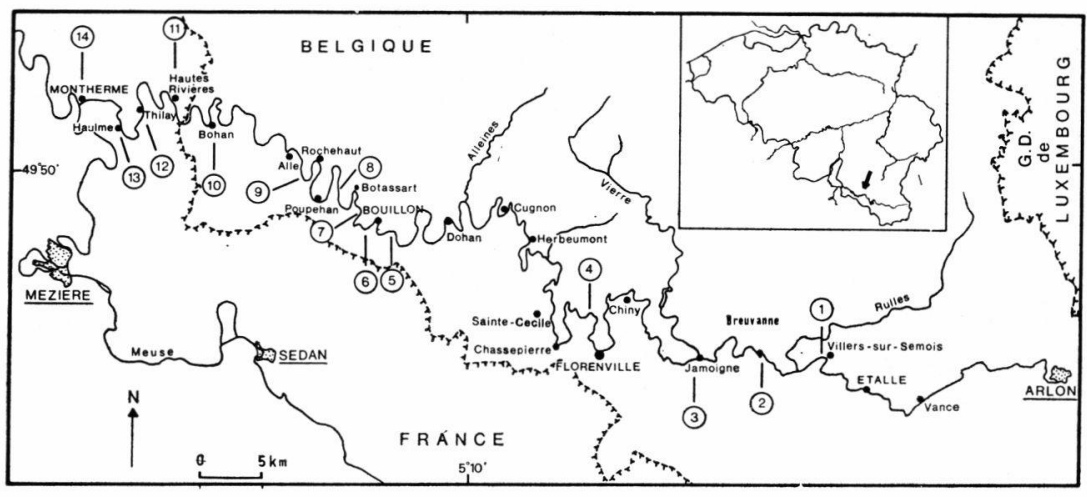

FIg. 1. - Topographie de la Semois et localisation des 8 stations étudiées. 9 et 10 : stations étudiées en 1959 par Huet et Timmermans (1963).

11 à 14: stations étudiées en juin 1977 par les services français de la Région piscicole des Ardennes (cfr F.U.L. 1977). 
lorraine et $1,13 \%$ en Semois ardennaise (Huet et Timmermans 1963), zonation piscicole (type inférieur de la Zone à Barbeau précédant le type supérieur: Huet et Timmermans 1963), composition chimique naturelle de l'eau (minéralisation, alcalinité et teneur en calcium diminuant de l'amont vers l'aval : Symoens 1957 ; Depasse 1977, F.U.L. 1977) et structure des communautés végétales (Symoens 1957, Pierre 1977, Descy 1977, Andrianne 1978).

Plusieurs études récentes (F.U.L. 1977 ; I.H.E. 1978) font le point sur les aspects physico-chimiques et certains aspects biologiques de la pollution organique et de l'eutrophisation dans l'ensemble de la Semois. De la source (égoûts de la ville d'Arlon) à Vance, la pollution organique est aiguë. Elle reste forte jusqu'au confluent de la Rulles. A partir de Breuvanne, la qualité de l'eau s'améliore et à l'aval de Chiny la pollution organique est résorbée. Dans le reste du cours et principalement en Basse Semois (aval de Bouillon) existe une eutrophisation sérieuse ; en 1975 et 1976, des "blooms " d'algues ont causé des mortalités massives de poissons en Basse Semois belge et surtout en Semoy française (Pierre 1977).

Le tableau I présente les principales caractéristiques physiographiques et physico-chimiques ainsi que les indices biotiques dans huit stations (st 1 à st 8 ) choisies pour effectuer les inventaires ichtyologiques. St 1 est située à la fin de la zone de forte pollution organique $(>0,025 \mathrm{mg} / 1 \mathrm{NH} 3$ non ionisée), St 2 et St 3 se trouvent dans la zone de régénération (rarement $>0,025 \mathrm{mg} / 1 \mathrm{NH} 3$ non ionisé) et les autres stations sont dans la zone eutrophisée.

\section{2. - METHODES}

Les inventaires piscicoles par pêche à l'électricité (courant continu : générateurs Electro Pulman 2,5 KWA et Szabo 1,4 et 0,8 KWA) furent réalisés en juillet-septembre 1977. Les secteurs échantillons $(85-255 \mathrm{~m}$; 0,055-0,73 ha) étaient barrés à l'amont au moyen d'un filet (st. 1 et 2) ou d'un écran électrifié (courant alternatif : générateur Honda $\mathrm{E} 400$ ) puis soumis à un effort intensif de pêche "à pied " en utilisant une (st 1 et 2), deux (st 3 et st 4) ou trois (st 5-8) anodes selon la largeur de la rivière. Tous les poissons capturés étaient identifiés, comptés, mesurés (longueur au creux de la nageoire caudale), éventuellement pesés et ensuite remis à l'eau.

La biomasse des captures par station est calculée grâce aux histogrammes des fréquences des longueurs (classes de 0,5 ou $1 \mathrm{~cm}$ ) et en utilisant les relations poids-longueur spécifiques établies pour les populations de l'Ourthe (Philippart $1977 \mathrm{~b}$ ). Les biomasses absolues des peuplements sont estimées par la méthode du coefficient d'effi- 
TABLEAU I. - Caractères phys:ographiques et physico-chimiques des stations de la Semois inventoriées par pêche à l'électricité en juillet-septembre 1977 (analyses chimiques au moyen de la trousse Hach le 23-09-1977 sauf celles des lignes notées (2) et (3)).

\begin{tabular}{|c|c|c|c|c|c|c|c|c|}
\hline \multirow{3}{*}{$\begin{array}{l}\text { Paramètres } \\
\text { Distance à la source } \\
\text { km }\end{array}$} & \multicolumn{8}{|c|}{ Stations } \\
\hline & st 1 & st 2 & st 3 & st 4 & st 5 & st 6 & st 7 & st 8 \\
\hline & 29 & 38 & 50 & 74 & 142 & 146 & 149 & 154 \\
\hline Région ${ }^{(1)}$ & H.S & M.S. & M.S. & M.S. & B.S. & B.S. & B.S. & B.S. \\
\hline Zone à Barbeau & \multicolumn{2}{|c|}{ type inférieur } & \multicolumn{6}{|c|}{ type supérieur } \\
\hline $\begin{array}{l}\text { Largeur } \\
\text { moyenne (m) }\end{array}$ & 6,5 & 17,1 & 26,8 & 20,0 & 45,5 & 26,3 & 32,5 & 40,0 \\
\hline Longueur (m) & 85 & 200 & 200 & 255 & 150 & 175 & 225 & 150 \\
\hline Superficie (Ha) & 0,055 & 0,345 & 0,535 & 0,510 & 0,680 & 0,460 & 0,730 & 0,600 \\
\hline Température $\left({ }^{\circ} \mathrm{C}\right)$ & 10,5 & 10,1 & 10,5 & 11,0 & 11,1 & 11,0 & 11,0 & 11,0 \\
\hline $\mathrm{pH}^{(2)}$ & 7,9 & 7,7 & 7,5 & 7,2 & - & - & - & 7,2 \\
\hline $\begin{array}{l}\text { Conductivité } \\
(\mu \mathrm{mhos} / \mathrm{cm})\end{array}$ & 410 & 265 & 270 & 240 & 191 & 196 & 189 & 187 \\
\hline $\begin{array}{l}\text { Alcalinité } \\
\left(\mathrm{mg} / \mathrm{l} \mathrm{CaCO}_{3}\right)\end{array}$ & 180 & 100 & 105 & 90 & 70 & 75 & 65 & 65 \\
\hline $\mathrm{Ca}^{++}(\mathrm{mg} / \mathrm{l})$ & 84 & 43 & 42 & 38 & 28 & 28 & 27 & 27 \\
\hline $\mathrm{NH}_{+}^{+}(\mathrm{mg} / \mathrm{l})^{(2)}$ & 6,1 & 1,5 & 0,5 & 0,2 & 一 & - & - & 0,2 \\
\hline $\mathrm{NC}_{2}^{-}(\mathrm{mg} / \mathrm{l})^{(2)}$ & 0,53 & 0,23 & 0,16 & 0,07 & - & - & - & 0,03 \\
\hline | & 7,3 & 7,5 & 7,7 & 1,5 & - & - & - & 5,0 \\
\hline $\mathrm{NU}_{3} \quad(\mathrm{mg} / 1)$ & 7,4 & 7,5 & 7,5 & 6,6 & 4,4 & 4,0 & 3,5 & 4,0 \\
\hline (E) & 7,0 & 1,5 & 0,9 & 0,6 & - & - & - & 5,0 \\
\hline $\mathrm{PO}_{*}(\mathrm{mg} / \mathrm{l})$ & 8,1 & 2,0 & 1,0 & 0,8 & 0,9 & 0,6 & 0,5 & 0,4 \\
\hline$\Leftrightarrow$ & 95 & 100 & 98 & 85 & - & - & - & 100 \\
\hline $\mathrm{O}_{2}$ (\% lot.) & 45 & 66 & 63 & 81 & - & - & - & (128) \\
\hline $\begin{array}{l}\text { Oxydabilité('(3) } \\
\quad\left(\mathrm{O}_{2} \mathrm{mg} / \mathrm{l}\right)\end{array}$ & 3,3 & 2,4 & 3,6 & 2,1 & - & 一 & - & $(1,3)$ \\
\hline Indice biotique (t) & 5,5 & 6,5 & 8,0 & 8,5 & 8,5 & - & - & 8,0 \\
\hline
\end{tabular}

(1) H.S. : Haute Semois ; M.S. : Moyenne Semois ; B.S. : Basse Semois.

(2) Mesures effectuées sur le terrain pendant la campagne de pêches par le $\mathrm{D}^{\mathrm{r}}$-Ing. J. Biernaux de la Faculté Agronomique de l'Etat à Gembloux.

(3) Mesures effectuées le 05-07-1977 par la F.U.L. ; les données pour la station 8 sont celles de Poupehan, situé quelques kilomètres à l'aval.

(4) Moyennes des analyses de mai, juillet et août 1977 (F.U.L., 1977).

cacité de pêche en un passage (Huet et Timmermans 1963, 1966); les coefficients d'efficacité (C. E.) sont déterminés par comparaison à l'efficacité réelle de pêche dans l'Ourthe (lors de dénombrements par marquage-recapture et efforts de capture successifs) et en tenant compte du nombre d'anodes de pêche en fonction de la largeur : C.E. $=0,45$ en st 1 et 0,65 dans les autres stations. 


\section{3. - RESULTATS}

Le tableau II indique le nombre de poissons de chaque espèce capturés à l'hectare dans les huit stations; la figure 2 donne les estimations de biomasse pour l'ichtyocénose et pour les groupes écologiques d'espèces selon la classification de Huet (1949).

Pour l'ensemble des stations, 1 espèce de Cyclostome et 19 espèces de Poissons Téléostéens ont été recensés.

Tableau II. - Abondance numérique (nombre/ha) des poissons et cyclostomes capturés par pêche à l'électricité dans 8 stations de la Semois en juillet-septembre 1977.

\begin{tabular}{|c|c|c|c|c|c|c|c|c|}
\hline Espèces & st 1 & st 2 & st 3 & st 4 & $\begin{array}{l}\text { ations } \\
\text { st } 5\end{array}$ & st 6 & st 7 & st 8 \\
\hline Salmo trutta fario & - & 12 & 4 & 55 & 6 & 15 & 14 & - \\
\hline Barbus barbus & - & 32 & 84 & 206 & 241 & 690 & 234 & 417 \\
\hline Leuciscus cephalus & 255 & 174 & 80 & 55 & 184 & 448 & 406 & 177 \\
\hline Chondrostoma nasus & - & - & 6 & 194 & 22 & 87 & 10 & 132 \\
\hline Leuciscus leuciscus & 55 & 17 & 127 & 123 & 85 & 492 & 229 & 377 \\
\hline Rutilus rutilus & - & - & 4 & 58 & 393 & 580 & 203 & 710 \\
\hline $\begin{array}{l}\text { Scardinius } \\
\quad \text { erythrophtalmus }\end{array}$ & - & - & 2 & - & - & - & - & - \\
\hline Gobio gobio & 272 & 620 & 246 & 67 & 478 & 274 & 235 & 147 \\
\hline Phoxinus phoxinus & 36 & - & - & 82 & 10 & 2 & 2 & 8 \\
\hline Alburnoides bipunctatus & - & - & - & 4 & 2 & 2 & 18 & - \\
\hline Alburnus alburnus & - & - & - & - & 16 & 148 & 62 & 20 \\
\hline Tinca tinca & - & 12 & 15 & - & 3 & 24 & - & 3 \\
\hline Abramis brama & - & - & - & - & - & 一 & - & 2 \\
\hline Esox lucius & 36 & 6 & 21 & - & 2 & 30 & 16 & 15 \\
\hline Perca fluviatilis & - & 9 & 11 & 16 & 7 & 52 & 21 & 8 \\
\hline Anguilla anguilla & 91 & 14 & 15 & 25 & 31 & 54 & 23 & 33 \\
\hline Cottus gobio & - & - & - & 2 & - & - & - & 3 \\
\hline Nemacheilus barbatulus & 55 & 31 & 6 & 27 & 63 & 4 & 4 & 8 \\
\hline Gasterosteus aculeatus & - & - & - & - & - & 2 & - & - \\
\hline Lampetra planeri & - & 3 & - & - & - & - & - & - \\
\hline Total & 800 & 930 & 621 & 914 & 1543 & 2904 & 1477 & 2060 \\
\hline
\end{tabular}

Sur la base des critères pente et largeur de Huet (1949), la totalité de la Semois est une Zone à Barbeau; l'ichtyocénose caractéristique de cette zone apparait dans les stations 3 à 8. Les cyprinidae rhéophiles lithophiles (Balon 1975) ou cyprins d'eau vive forment $69,2-84,5 \%$ de l'ichtyomasse; au sein de ce groupe $B$. barbus est dominant (38,3$49,7 \%$ ) et représente une fraction relativement constante. $L$. cephalus $(56,2-23,3 \%), C$. nasus $(5,3-30,6 \%)$ et L. leuciscus $(1,0-14,2 \%)$ ont des 


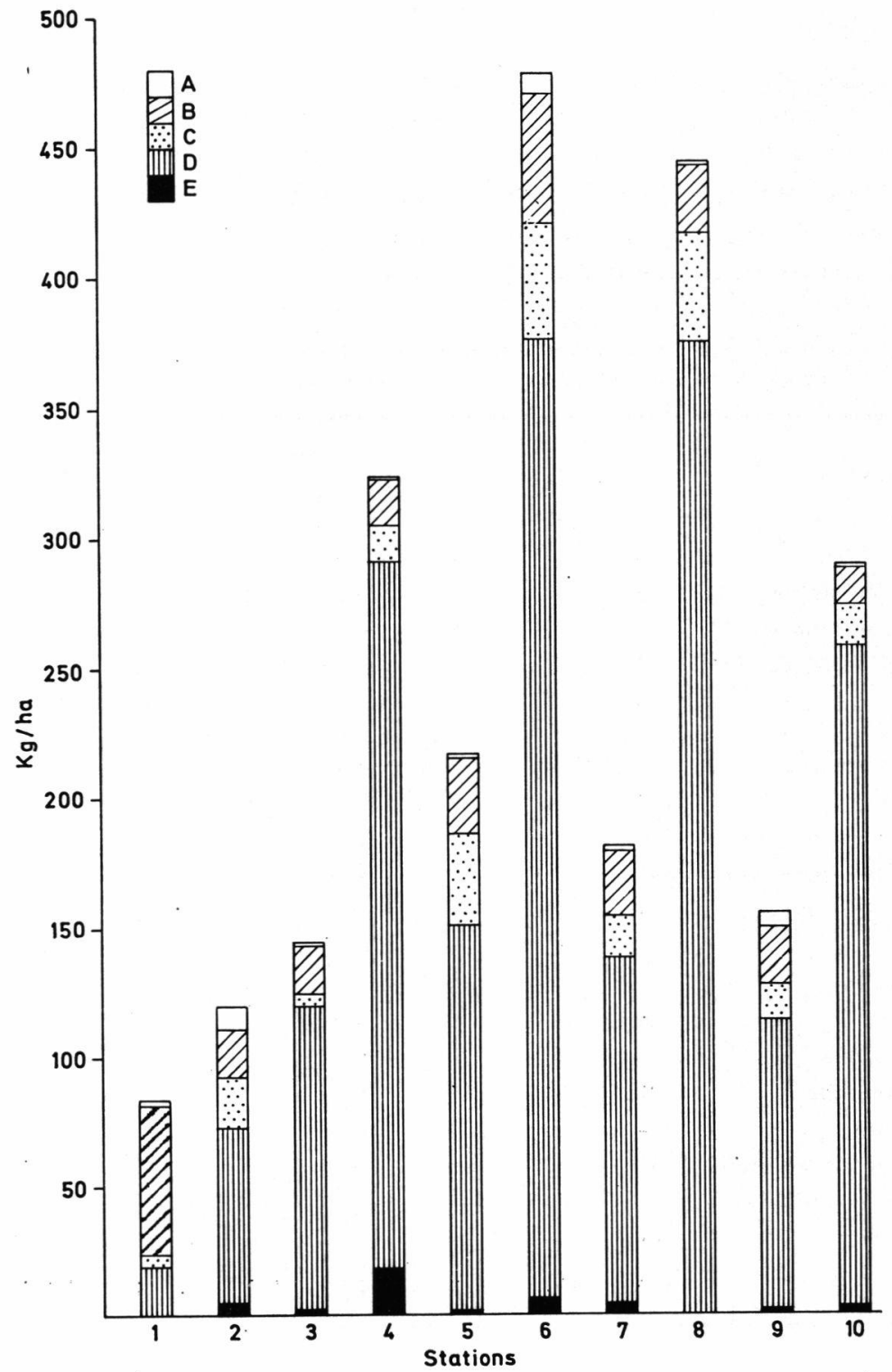

Fig. 2. - Biomasse absolue de l'ichtyocénose et des principaux groupes écologiques d'espèces dans la Semois en 1977. Comparaison aux résultats de Huet et Timmermans (1963) pour les stations d'Alle (9) et Bohan (10) en 1959.

A : cyprins d'eau calme ( $T$. tinca, A. brama) et autres espèces.

B : prédateurs d'accompagnement (E. lucius, $P$. fluviatilis, A. anguilla).

C : cyprins d'accompagnement (R. rutilus, S. erythrophtalmus, G. gobio).

D : cyprins d'eau vive (B. barbus, C. nasus, L. cephalus, L. leuciscus).

E : salmonides (S. trutta). 
peuplements beaucoup plus variables selon les stations. Les cyprins d'accompagnement contribuent à $0,6-16,3 \%$ de l'ichtyomasse, $R$. rutilus intervenant pour la plus grande part $(0,5-11,5 \%)$. Les prédateurs d'accompagnement représentent 3,7-13,6\% de l'ichtyomasse pour 0,0$5,7 \%$ pour l'unique salmonide, $S$. trutta et moins de $1,5 \%$ pour les cyprins d'eau calme. Vu les conditions d'échantillonnage, il est difficile d'interpréter biologiquement les différences entre les stations; notons cependant que $R$. rutilus est proportionnellement plus abondant que L. leuciscus dans la Basse Semois (st 5-8) par rapport à la Moyenne Semois (st 4) où la pente est plus forte.

L'ichtyofaune type de la Basse Semois belge caractérise également le début de la Semoy française (st 10 : les cyprins d'eau vive et $B$. barbus forment respectivement $74 \%$ et $52,4 \%$ de l'ichtyomasse ; F. U.L. 1977) ; théoriquement, elle devrait aussi se retrouver dans le reste de la Semoy et dans les stations (st 1 et st 2) de Haute et de Moyenne Semois. La discordance entre l'ichtyocénose potentielle ct les résultats des inventaires mesure les altérations subies aux différents points.

A la station très polluée st 1 , l'ichtyofaune est anormale à tous points de vue : faible diversité spécifique (7 spp.), absence des espèces sensibles ( $C$. gobio, $S$. trutta et $C$. nasus), déséquilibre affectant la biomasse relative des espèces et groupes d'espèces (importance réduite des cyprins d'eau vive et d'accompagnement, abondance d'A. anguilla et d'E. lucius, biomasse absolue $-83 \mathrm{~kg} / \mathrm{ha}-3-4$ fois moindre que celle attendue compte tenu de la fertilité naturelle de l'eau). En st 2 , la régénération de la rivière se marque par l'augmentation du nombre d'espèces (11 spp.), la présence de $S$. trutta et $B$. barbus, l'apparition d'une ichtyocénose mieux équilibrée $(4,0 \%$ de l'ichtyomasse en salmonides et $56 \%$ en cyprins d'eau vive) et l'augmentation de l'ichtyomasse $(120 \mathrm{~kg} / \mathrm{ha})$; mais l'altération structurelle de l'ichtyocénose reste importante et s'exprime par l'absence de $C$. gobio et $C$. nasus et par la prépondérance de $L$. cephalus $(47,7 \%)$ sur $B$. barbus. En st 3, la composition par espèces (réapparition de $C$. nasus) et leurs biomasses relatives (rétablissement de la prépondérance de $B$. barbus sur $L$. cephalus) redeviennent normales mais l'ichtyomasse globale est faible (145 kg/ha) par rapport à Martué (st $4: 325 \mathrm{~kg} / \mathrm{ha}$ ) qui marque le retour à une situation équilibrée.

En Basse Semois (st 5-st 8), l'eutrophisation du milieu se manifeste par l'absence ou la rareté des espèces oxyphiles comme $C$. gobio et $S$. trutta et d'A. bipunctatus. La rareté de $P$. phoxinus (et de $S$. trutta dans certaines stations) provient davantage de la surexploitation halieutique.

L'analyse des données disponibles pour la Semoy (F.U.L. 1977) met en évidence de l'amont vers l'aval (st 11 à st 14) une altération struc- 
turelle croissante de l'ichtyocénose qui se présente sous la même forme que celle observée quand on remonte de st 4 en st 1 : substitution progressive de $L$. cephalus à $B$. barbus, rareté ou absence totale de $C$. nasus, réduction de l'abondance relative des cyprins d'eau vive ( $L$. cephalus et $B$. barbus en st 11,12 et 13 et $L$. cephalus seul en st 14) au profit des autres cyprins ( $G$. gobio et $R$. rutilus) et des prédateurs (E. lucius, $P$. fluviatilis et $A$. anguilla).

En conclusion, les deux types de pollution (organique et chronique en Haute Semois ; "accidentelle " et due à une floraison d'algues en Semoy) opèrent par exclusion ou mortalité différentielle des espèces selon une polluosensibilité (Verneaux 1976) décroissant dans l'ordre suivant : C. gobio - S. trutta - A. bipunctatus - C. nasus - B. barbus L. leuciscus - L. cephalus. En outre, d'après nos observations, la biomasse relative du groupe $B$. barbus - $C$. nasus s'avère un bon indice pour évaluer le degré d'équilibre de l'ichtyocénose d'une Zone à Barbeau. Dans une Zone à Barbeau non polluée et non altérée physiquement (Ourthe: Philippart 1977 b; Jihlava : Lusk 1977), ce groupe forme $50-70 \%$ de l'ichtyomasse. Dans la Semois, l'utilisation de cet indice permet de localiser ( $f$ ig. 3) les tronçons altérés de l'amont et de l'aval et la zone relativement peu altérée entre Martué (st 1) et la frontière.

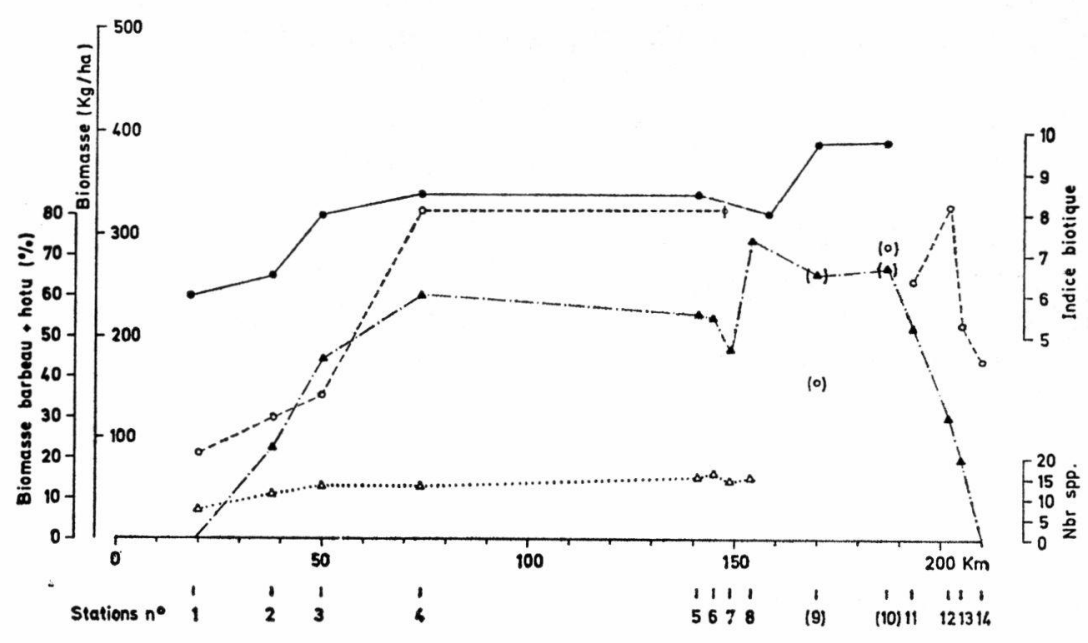

Fig. 3. - Profil longitudinal des principaux caractères de l'ichtyofaune et des indices biotiques dans la Semois en 1977.

Cercle noir: Indices biotiques selon Tuffery et Verneaux (F.U.L., 1977).

Triangle blanc: Nombre d'espèces de poissons téléostéens.

Cercle blanc: Biomasse absolue de l'ichtyocénose $(\mathrm{Kg} / \mathrm{Ha})$ ( $\varnothing$ moyenne St 5St 8).

Triangle noir: Biomasse relative du groupe B. barbus - $C$. nasus (en \% de l'ichtyomasse totale).

() Situation 1959 (Huet et Timmermans 1963). 
On notera enfin sur le graphique de synthèse de la fig. 3 la concordance entre les profils ichtyologiques et le profil des indices biotiques basés sur l'analyse des biocénoses d'Invertébrés benthiques.

\section{4. - DISCUSSION ET CONCLUSIONS GENERALES}

Par rapport aux inventaires réalisés en Basse Semois en 1959 (Huet et Timmermans 1963) nos résultats (st 5-st 8) ne révèlent aucune modification majeure de la biomasse absolue des peuplements et de l'abondance relative des espèces et groupes d'espèces de grande taille ( $f$ ig. 2 et 3) Les différences mineures observées peuvent s'expliquer par les variations dues à l'échantillonnage ou par les fluctuations annuelles du recrutement des classes d'âge. En fait, l'évolution de la faune ichtyologique de la Basse Semois belge au cours des vingt dernières années s'est surtout manifestée par la régression des espèces de petite taille ( $P$. phoxinus, $C$. gobio, A. alburnoides). Ces trois espèces sembiaient encore assez abondantes en 1971 (Philippart 1971) et leur raréfaction apparaît comme un phénomène relativement récent (5 ans) qu'on peut associer au dépassement d'un niveau critique d'eutrophisation, visualisé en 1975 et 1976 par la mortalité des espèces de grande taille en Semoy. Vu la situation actuelle, une dégradation plus grave de l'ichtyocénose de la Basse Semois est à craindre si des mesures efficaces d'épuration ne sont prises pour stabiliser cette eutrophisation et à moyen terme pour la réduire.

Les deux types de pollution analysés dans cette étude se traduisent au niveau de l'ichtyocénose par les formes d'altération classiques des biocénoses aquatiques (Tuffery 1976, Verneaux 1976, Hawkes 1977) : réduction de la richesse spécifique par élimination des espèces les plus sensibles, réduction de l'abondance (biomasse) relative des espèces sensibles au profit des espèces tolérantes avec parfois remplacement des unes par les autres, diminution de la biomasse absolue de lichtyocénose. Le caractère "accidentel " des pollutions en Semoy soulève en outre le problème de la recolonisation différentielle de la rivière; sur ce plan, l'exemple de l'Amblève (fig. 4) permet de supposer pour la Semoy la reconstitution d'une communauté différente de la communauté initiale, (par ex.: substitution de $L$. cephalus à $B$. barbus et dans une moindre mesure à $C$. nasus). Ces différentes formes de réponse des populations et communautés de poissons aux "stress » eutrophisation et pollution font intervenir des processus de nature écophysiologique (besoin en oxygène, tolérance thermique, sensibilité à NH3 ou aux autres toxiques chimiques), démographique (potentiel de reproduction, résilience) et biocénotique (compétition interspécifique) encore peu connus pour la plupart des espèces européennes, notamment les cyprinidae. En ce qui concerne $B$. barbus, 

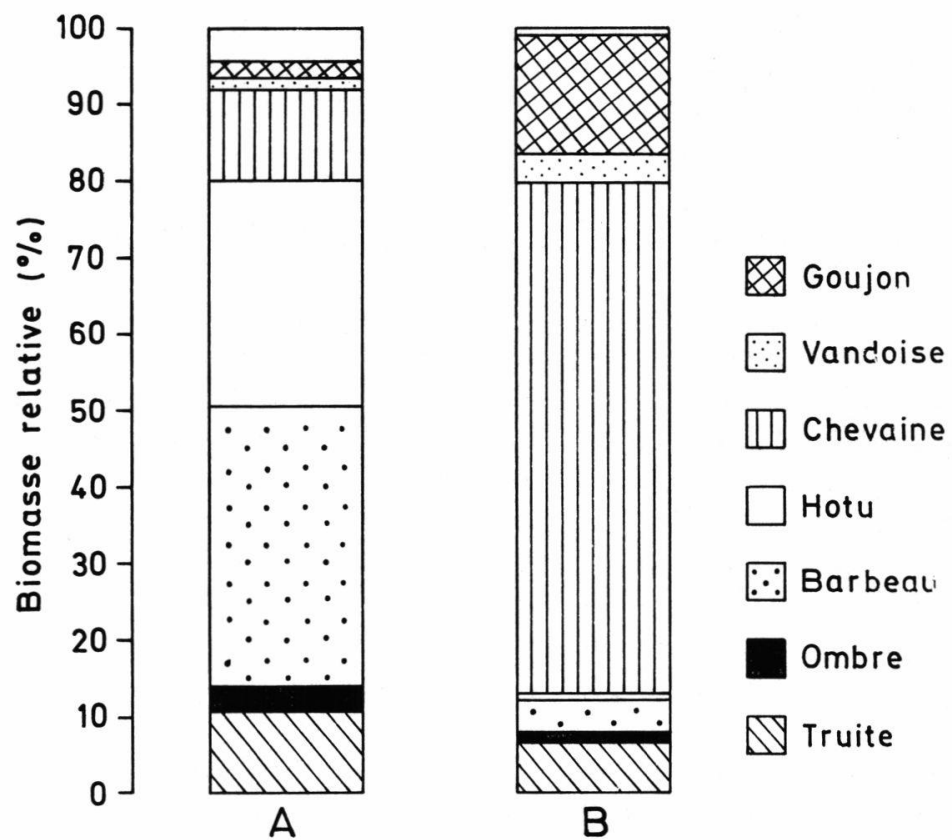

Fig. 4. - Exemple de modification structurelle d'une ichtyocénose suite à la recolonisation du milieu après une pollution.

A. structure normale dans une Zone à Ombre (type supérieur) (Ourthe de Maboge : Huet et Timmermans, 1966).

B. structure déséquilibrée dans l'Amblève 10 ans après une pollution totale.

la forte sensibilité de ses populations aux altérations de la qualité de l'eau pourrait être associée à la longueur (11-19 jours pour 10 jours chez $L$. cephalus) de la phase éleuthéro-embryonnaire de nutrition vitelline et de vie passive sur le fond (Penaz 1973) ; cette hypothèse permet notamment de comprendre le remplacement de B. barbus par L. cephalus dans les rivières polluées. $B$. barbus présente d'autre part des caractéristiques démographiques d'espèce à stratégie $-\mathrm{K}$ (maturité sexuelle tardive des femelles, faible fécondité relative: Philippart 1977 b) ; cet élément expliquerait sa difficulté à reconstituer ses populations après une pollution aiguë et son incapacité à maintenir des populations stables dans des milieux où les conditions physico-chimiques compromettent la survie des alevins et limitent les peuplements en-dessous d'une valeur biologiquement minimale pour la reproduction.

En conclusion générale de cette étude de cas sur la faune ichtyologique de la Semois, il apparaît indispensable d'approfondir les connaissances sur les aspects écophysiologiques et démographiques de la sensibilité différentielle des espèces de poissons aux multiples formes de pollution et de perturbations du milieu aquatique (travaux, 
pêche, etc...). A cet égard, plusieurs espèces sauvages méritent une attention particulière (ex.: B. barbus parmi les Cyprinidae et $T$. thymallus parmi les Salmonidae) à cause de la régression de leurs populations dans de nombreuses rivières; cette situation exige des mesures de conservation et de protection qui impliquent l'identification exacte des causes de régression et l'évaluation des besoins écologiques précis de ces espèces (définition de normes spécifiques de qualité d'eau et du milieu, Philippart 1979). C'est un des objectifs de nos recherches actuelles sur l'écologie comparée des populations de poissons (principalement cyprins rhéophiles) dans les rivières du bassin de la Meuse en Belgique.

\section{REMERCIEMENTS}

Les différentes phases de cette étude ont pu être réalisées grâce au concours de nombreuses personnes et institutions. Nous tenons spécialement à remercier l'Administration des Eaux et Forêts (permis de pêche et participation aux pêches électriques), la Fondation Universitaire Luxembourgeoise (appui technique et logistique lors des pêches de juillet 1977), la Fédération de Pêche de la BasseSemois et notamment la Société de Bouillon (autorisation de pêche et appui logistique).

Les études sur la Semois s'intégraient dant un programme de recherches entrepris grâce au support financier du Fonds National de la Recherche Scientifique (mandat de recherche et crédit de recherche $\mathrm{n}^{\circ}$ S 2/5-FG-88110-D) et de la Commission provinciale de Liège du Fonds Piscicole (subside de fonctionnement) et avec l'appui logistique et en personnel de l'Aquarium de l'Université de Liège $\left(D^{r} \mathrm{~J}\right.$. Voss) et du Service d'Ethologie et de Psychologie animales (Prof. J. C. Ruwet).

\section{TRAVAUX CITÉS}

ANDRIANNE (C.). 1978. - Etude de la végétation macrophytique de la Semois belge. Mise au point et application d'une méthode d'échantillonnage quantitative des algues macroscopiques. Mémoire de Licence en Sciences Botaniques. Université de Liège, 121 pages.

Baton (E. K.). 1975. - Reproductive guilds of fishes; a proposal and definition. J. Fish. Res. Board Canada, 32, 821-864.

Depasse (P.). 1977. - Où en est la pollution de la Semois ? Situation comparée 1959-1975. Prévisions. Les Naturalistes belges, 58, 119-132.

Descy (J. F.). 1977. - In Rapport F.U.L. (1977), Fascicule 4. Etude préliminaire des diatomées, 9 pages.

F.U.L. 1977. - Rapport d'activité relatif au contrat passé entre les Communautés Economiques Européennes et la Fondation Universitaire Luxembourgeoise (F.U.L.). Etat d'avancement au 31 décembre 1977. Fascicule 3: Etude de la Semois (Semoy) franco-belge: Eutrophisation, pages.

HAwkes (H. A.). 1977. - Biological classification of rivers: conceptual basis and ecological validity, p. 55-67. In J. S. Alabaster (Ed.), Biological monitoring of inland fisheries. Applied Science Publishers, London, 226 pages.

Huer (M.). 1949. - Aperçu des relations entre la pente et les populations piscicoles des eaux courantes. Revue Suisse d'Hydrologie, vol. XI (3-4), 332-351. 
Huet (M.) et Timmermans (J. A.). 1963. - La population piscicole de la Semois inférieure, grosse rivière belge du type supérieur de la Zone à Barbeau. Trav. Stat. Rech. Eaux et Forêts, série D, no 36, 1-31.

Huet (M.) et Timmermans (J. A.). 1966. - La population piscicole de l'Ourthe (grosse rivière belge de la Zone à Ombre et du type supérieur de la Zone à Barbeau). Verh. Internat. Verein. Limnol., 16, 1192-1203.

HolcrK (J.) et BaSTL (I.). 1976. - Ecological effects of water level fluctuation upon the fish populations in the Danube River flood-plain in Czechoslovakia. Acta Sc. Nat. Brno, 10 (9), 1-46.

I.H.E. 1978. - Aperçu quantitatif de quelques eaux superficielles belges en 1977. Ministère de la Santé Publique et de la Famille, Institut d'Hygiène et d'Epidémiologie (I.H.E.), Section Eau, 204 pages.

Kolder (W.), SKora (S.) et Wlodek (J.). 1974. - Ichtyofauna of the River Raba and its tributaries. Acta hydrobiol., 16, 65-99.

LUSK (S.). 1973. - The ichtyofauna of the Rokytna River. I. Species composition, abundance and biomass. Zool. Listy, 22, 165-180.

LUSK (S.). 1977. - The ichtyofauna of the middle course of the Jihlava River in relation to the water reservoir at Dalesice. Folia Zool. Brno, 26 (3), 255-269.

Micha (J. C.). 1971. - Etude des communautés piscicoles dans l'Ourthe liégeoise. Tribune du Cebedeau, 326, 1-7.

Penaz (M.). 1973. - Embryonic development of the barb, Barbus barbus (Linnaeus, 1758), Zool. listy, 22 (4), 363-374.

Penczak (T.) et Zalewski (M.). 1974. Distribution of fish number and biomass in barbel-region of the Pilica River and the adjoining old river-beds. Ekol. pol., 22, 107-119.

Penczak (T.), Zalewski (M.) et Molinski (M.). 1976. - Production of pike, roach and chub in a selected fragment of Pilica River. Pol. Arch. Hydrobiol., 23 (1), 139-153.

Penczak (T.). 1972. - Structure of fish grouping in the rivers and streams of the river Nida drainage basin. Ekol. pol., 20 (26), 1-18.

PhilipPaRT (J.C.). 1971. - Projet d'une étude piscicole de la Semois. Rapport sur les sondages piscicoles réalisés en octobre 1971. Rapport inédit, Université de Liège, 25 pages.

Philippart (J.C.). 1977 a. - Contribution à l'étude de l'écosystème « Rivière de la Zone à Barbeau supérieure ": Densité, biomasse et production des populations de poissons de l'Ourthe, pp. 551-567. In P. Duvigneaud et P. Kestemont (Ed.), Productivité biologique en Belgique, Duculot, Gembloux, Paris, 617 pages.

Philippart (J. C.). 1977 b. - Contribution à l'hydrobiologie de l'Ourthe. Dynamique des populations et production de quatre espèces de poissons cyprinidae. Thèse de Doctorat en Sciences Zoologiques, Université de Liège, 225 pages.

Philippart (J. C.). 1979. - Evaluation des ressources piscicoles et halieutiques dans les rivières du bassin de la Meuse, pp. 481-495. In L. Calembert (Ed.), Problématique et Gestion des Eaux Intérieures, Actes du Colloque de Liège, 16-19 mai 1978, Editions Derouaux, Liège, 967 pages.

Pierre (J. F.). 1977. - Algues et mortalité piscicole en Semois. La technique de l'eau et de l'assainissement, $\mathrm{n}^{\circ} 368-369,19-31$.

Roux (A. L.). 1976. Structure et fonctionnement des écosystèmes du Haut-Rhône français. I. Présentation de l'étude. Bull. Ecol., 7 (4), 479-492.

Sedlar (J.). 1969. - Die gegenwartige Beschaffenfeit des Fischbestandes im Einzugsgebiet des Flusses Nitra (Slovakei). Biol. Prace, 15 (2), 5-78.

Symorins (J. J.). 1957. - Les eaux douces de l'Ardenne et des régions voisines. Les milieux et leur végétation algale. Bull. Soc. Roy. Botan. Belgique, 89, 111-314. 
TUFFERY (G.). 1976. - Incidence écologique de la pollution des eaux courantes. Révélateurs biologiques de la pollution, p. 183-219. In P. Pesson (Ed.), La pollution des eaux continentales. Incidence sur les biocénoses aquatiques, Gauthier-Villars, Paris, 285 pages.

Vereerstraeten (J.). 1977. - Hydrogéographie quantitative de la Semois. Industries et Sciences, 2, 1-10.

Verneaux (J.). 1973. - Cours d'eau de Franche-Comté (massif du Jura). Recherches écologiques sur le réseau hydrographique du Doubs. Essai de biotypologie. Thèse de Doctorat ès Sciences Naturelles, Univ. Besançon, 257 pages.

Verneaux (J.). 1976. - Fondements biologiques et écologiques de l'étude de la qualité des eaux continentales. Principales méthodes biologiques, p. 229285, in P. Pesson (Ed.), La pollution des eaux continentales. Incidences sur les biocénoses aquatiques, Gauthier-Villars, Paris, 285 pages. 